J. Noncommut. Geom. 5 (2011), 591-614

DOI $10.4171 / \mathrm{JNCG} / 88$
Journal of Noncommutative Geometry

(C) European Mathematical Society

\title{
Piecewise principal comodule algebras
}

\author{
Piotr M. Hajac, Ulrich Krähmer, Rainer Matthes and Bartosz Zieliński
}

\author{
Dedicated to Hans-Jürgen Schneider \\ on the occasion of his 65th birthday
}

\begin{abstract}
A comodule algebra $P$ over a Hopf algebra $H$ with bijective antipode is called principal if the coaction of $H$ is Galois and $P$ is $H$-equivariantly projective (faithfully flat) over the coaction-invariant subalgebra $P^{\mathrm{co} H}$. We prove that principality is a piecewise property: given $N$ comodule-algebra surjections $P \rightarrow P_{i}$ whose kernels intersect to zero, $P$ is principal if and only if all $P_{i}$ 's are principal. Furthermore, assuming the principality of $P$, we show that the lattice these kernels generate is distributive if and only if so is the lattice obtained by intersection with $P^{\text {co } H}$. Finally, assuming the above distributivity property, we obtain a flabby sheaf of principal comodule algebras over a certain space that is universal for all such $N$-families of surjections $P \rightarrow P_{i}$ and such that the comodule algebra of global sections is $P$.
\end{abstract}

Mathematics Subject Classification (2010). 58B32.

Keywords. Principal actions of quantum groups, pullback diagrams.

\section{Introduction}

Comodule algebras provide a natural noncommutative-geometry generalisation of spaces equipped with group actions. Less evidently, principal extensions [BH04] appear to be a proper analogue of principal bundles in this context. Principal comodule algebras can be considered as functors from the category of finite-dimensional corepresentations of the Hopf algebra (replacing the structure group) to the category of finitely generated projective modules over the coaction-invariant subalgebra (playing the role of the base space). Thus we have a noncommutative version of assigning to a group representation an associated vector bundle. A precise statement expressing this in the setting of topological vector bundles can be found in [BHMS], Theorem 3.3.

The aim of this article is to establish a viable concept of locality of comodule algebras, and to analyse its relationship with principality. The notion of locality we use herein results from decomposing algebras into "pieces", meaning expressing them as multiple fibre products (called multirestricted direct sums in [P-GK99], p. 264). If $X$ is a compact Hausdorff space and $X_{1}, \ldots, X_{N}$ form a finite closed covering, then 
$C(X)$ can be expressed as such a multiple fibre product of its quotient $\mathrm{C}^{*}$-algebras $C\left(X_{i}\right)$. This leads to a $\mathrm{C}^{*}$-algebraic notion of a "covering of a quantum space" given by a finite family of algebra surjections $\pi_{i}: P \rightarrow P_{i}$ with $\bigcap_{i} \operatorname{ker} \pi_{i}=0$. (See [BK96], [CM00], cf. [D-M97].)

Recall that not all properties of group actions are local in nature: there is a natural example of a locally proper action of $\mathbb{R}$ on $\mathbb{R}^{2}$ that is not proper ([P-RS61], p. 298, [BHMS], Example 1.14). On the other hand, a group action is free if and only if it is locally free. Therefore, since for compact groups all actions are proper, the principal (i.e., free and proper) actions of compact groups are local in nature. Our main result is a noncommutative analogue of this statement.

Theorem 3.3. A comodule algebra $P$ "covered by pieces" $P_{i}$ is principal if and only if so are the pieces.

In particular, a smash product of an $H$-module algebra $B$ with the Hopf algebra $H$ (with bijective antipode) is principal so that gluing together smash products is a way of constructing principal comodule algebras. This is a major application of our result, and is presented in the example section. Another application of Theorem 3.3 was found recently in algebraic topology. Notably, it allows one to prove a compactgroup version of the well-known equivalence of discrete-group principal bundles and Galois covering maps $[\mathrm{BH}]$.

More precisely, first one extends the notion of the algebra of regular functions (spanned by the matrix coefficients of the irreducible unitary corepresentations) from compact quantum groups to unital $C^{*}$-algebras on which they act. Such a subalgebra of regular functions is called the Peter-Weyl comodule algebra. The history of this concept and its fundamental relationship to the canonical decomposition of a compactgroup representation into isotypical components is explained in [S-PM]. The PeterWeyl algebra is a comodule algebra over the Hopf algebra of regular functions on a compact quantum group. In general, it is not a $\mathrm{C}^{*}$-algebra, although its coactioninvariant (the base space) subalgebra is always a $\mathrm{C}^{*}$-algebra. Now, one can show that the Peter-Weyl comodule algebra of functions on a compact Hausdorff space with an action of a compact group is principal if and only if the action is free [BH]. In other words, the Galois condition of Hopf-Galois theory holds if and only if we have a compact principal bundle.

Concerning coverings of a quantum space in the earlier described sense, it was pointed out in [CM00], p. 369, that they feature a certain incompleteness problem when going beyond closed ideals of $\mathrm{C}^{*}$-algebras. This is related to the fact that the lattice of ideals generated by the ker $\pi_{i}$ 's is in general not distributive. Hence we analyse a stronger notion of a covering that includes the assumption of distributivity as part of Definition 3.6. This allows us to consider well-behaved coverings beyond the $\mathrm{C}^{*}$-setting, which is useful for Peter-Weyl comodule algebras. Furthermore, if all covering comodule algebras are smash products, we arrive at a concept of piecewise trivial comodule algebras. They appear to be a good noncommutative replacement 
of locally trivial compact principal bundles.

The data of a covering by $N$ pieces can be equivalently encoded into a flabby sheaf of algebras over $\mathbb{P}^{N-1}(\mathbb{Z} / 2)$. This is the 2-element field $(N-1)$-projective space whose topology subbasis is its usual covering by $N$ affine spaces. It is a finite space encoding the combinatorics of an $N$-covering, and is non-Hausdorff unless $N=1$. The family of all non-empty open subsets of $\mathbb{P}^{N-1}(\mathbb{Z} / 2)$ turns out to be a lattice isomorphic to the free distributive lattice on $N$ generators. ${ }^{1}$ Combining this with the Chinese Remainder Theorem for distributive lattices of ideals in an arbitrary ring proves that distributive lattices generated by $N$ ideals are equivalent to flabby sheaves over $\mathbb{P}^{N-1}(\mathbb{Z} / 2)$ (cf. Lattice of Ideals in [R-GC97]).

For example, consider a compact Hausdorff space $X$ with a covering by $N$ closed subsets $X_{1}, \ldots, X_{N}$. Then we have the soft sheaf of continuous functions with $N$ distinguished $\mathrm{C}^{*}$-algebras $C\left(X_{1}\right), \ldots, C\left(X_{N}\right)$. However, the soft sheaf of complexvalued continuous functions on $X$ is not a sheaf of $\mathrm{C}^{*}$-algebras. Therefore, there seems to be no evident way to use soft sheaves in the noncommutative setting. To overcome this difficulty, we declare the closed sets open and consider $X$ with the new topology generated by these open sets. This leads us to flabby sheaves over $\mathbb{P}^{N-1}(\mathbb{Z} / 2)$.

Thus we arrive at a covering version of the commutative Gelfand-Naimark Theorem: There is an equivalence between the category of compact Hausdorff spaces with ordered closed coverings by $N$ subsets and the opposite category of flabby sheaves of unital commutative $\mathrm{C}^{*}$-algebras over $\mathbb{P}^{N-1}(\mathbb{Z} / 2)$. In the noncommutative setting, this sheaf-theoretic reformulation of coverings allows us to view a piecewise trivial comodule algebra as a geometrically desired refinement of what is called an "A-quantum principal bundle" in [P-MJ94]. In particular, our framework allows us to conclude that the comodule algebra of global sections is principal.

Summarising, much as the gluings of topological spaces, the pullbacks of $\mathrm{C}^{*-}$ algebras are fundamental for constructing new algebras and applying Mayer-Vietoris arguments. We are motivated by the study of pullbacks of $\mathrm{C}^{*}$-algebras equipped with a quantum group action. These pullbacks can be exemplified by a join construction of compact quantum groups. Insisting on the freeness of these actions leads to Galoistype extensions of $\mathrm{C}^{*}$-algebras by Hopf algebras. This allows us to use at the same time both algebraic techniques of Hopf-Galois theory and analytic tools coming with $\mathrm{C}^{*}$-algebras. Generalising pullbacks from two to finitely many morphisms brings up distributive lattices as a fundamental language. As a by-product of our considerations, we obtain an equivalence between the distributive lattices generated by $N$ ideals intersecting to zero and flabby sheaves of algebras over a projective space $P^{N-1}(\mathbb{Z} / 2)$.

${ }^{1}$ Note that it is extremely difficult to determine the number of elements $F D(N)$ of this finite lattice. The asymptotic behaviour of $F D$ was analysed in [Y-K54], but the problem of finding a general formula for $F D(N)$, originally posed by R. Dedekind [D-R97], p. 147, remains open since 1897. In 1999, the biggest known value of $F D$ was for $N=8$. 


\section{Background}

Throughout, we work over a field $k$. All considered algebras, coalgebras etc., are over $k$. An unadorned $\otimes$ denotes the tensor product of $k$-vector spaces. For coproducts and coactions we adopt the Heyneman-Sweedler notation with the summation sign suppressed: $\Delta(h)=h_{(1)} \otimes h_{(2)} \in H \otimes H, \Delta_{P}(p)=p_{(0)} \otimes p_{(1)} \in P \otimes H$.

2.1. Fibre products. We recall here elementary facts concerning pullback diagrams that will be used in what follows. To focus attention, we consider the category of vector spaces. Since all our algebras and comodules are over a field, this suffices for our applications.

Let $\pi_{1}: V_{1} \rightarrow V_{12}$ and $\pi_{2}: V_{2} \rightarrow V_{12}$ be linear maps of vector spaces. The pullback (fibre product) $V_{1} \times_{\pi_{1}, \pi_{2}} V_{2}$ of $\pi_{1}$ and $\pi_{2}$ is defined by a universal property, and turns out to be isomorphic to $\operatorname{ker}\left(\pi_{1}-\pi_{2}: V_{1} \times V_{2} \rightarrow V_{12}\right)=\left\{(p, q) \in V_{1} \times V_{2} \mid\right.$ $\left.\pi_{1}(p)=\pi_{2}(q)\right\}$. As a consequence of this description, we obtain

$$
\left(V_{1} \times_{\pi_{1}, \pi_{2}} V_{2}\right)^{\otimes 2}=\operatorname{ker}\left(\left(\pi_{1}-\pi_{2}\right) \otimes \mathrm{id}\right) \cap \operatorname{ker}\left(\mathrm{id} \otimes\left(\pi_{1}-\pi_{2}\right)\right) .
$$

Next, let us consider the following commutative diagram of linear maps

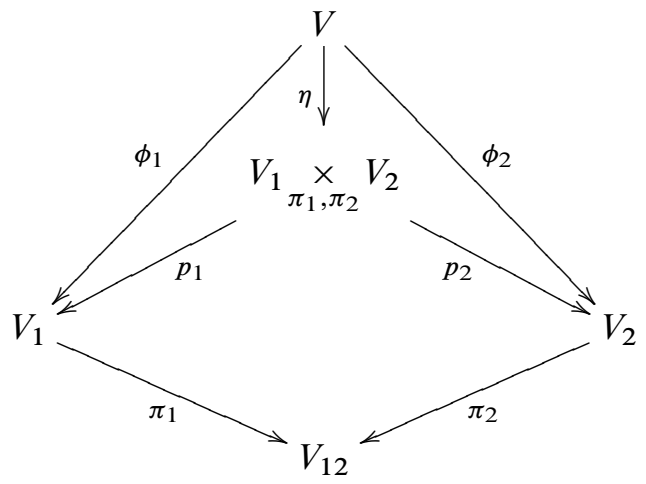

and unravel the structure of their kernels.

Lemma 2.1. Assume that the $\phi_{i}$ 's and $\pi_{i}$ 's in the diagram (2) are surjective. Then $\eta$ is surjective if and only if $\operatorname{ker}\left(\pi_{i} \circ \phi_{i}\right)=\operatorname{ker} \phi_{1}+\operatorname{ker} \phi_{2}$.

Proof. Assume first that $\eta$ is surjective and $v \in \operatorname{ker}\left(\pi_{i} \circ \phi_{i}\right)$. Then both $\left(\phi_{1}(v), 0\right)$ and $\left(0, \phi_{2}(v)\right)$ belong to $V_{1} \times_{\pi_{1}, \pi_{2}} V_{2}$, and there exist $v_{1}$ and $v_{2}$ such that $\eta\left(v_{1}\right)=$ $\left(\phi_{1}(v), 0\right)$ and $\eta\left(v_{2}\right)=\left(0, \phi_{2}(v)\right)$. Clearly, $v-\left(v_{1}+v_{2}\right) \in$ ker $\eta$. Therefore, as $v_{1} \in \operatorname{ker} \phi_{2}, v_{2} \in \operatorname{ker} \phi_{1}$, and

$$
\operatorname{ker} \eta=\operatorname{ker} \phi_{1} \cap \operatorname{ker} \phi_{2},
$$

we conclude that $v \in \operatorname{ker} \phi_{1}+\operatorname{ker} \phi_{2}$. 
Conversely, assume that $\operatorname{ker}\left(\pi_{i} \circ \phi_{i}\right)=\operatorname{ker} \phi_{1}+\operatorname{ker} \phi_{2}$. Let $\left(\phi_{1}\left(v_{1}\right), \phi_{2}\left(v_{2}\right)\right)$ be any element of the fibre product. Then $v_{1}-v_{2} \in \operatorname{ker} \phi_{1}+\operatorname{ker} \phi_{2}$ so that $v_{1}-v_{2}=$ $k_{1}+k_{2}$ for some $k_{1} \in \operatorname{ker} \phi_{1}$ and $k_{2} \in \operatorname{ker} \phi_{2}$. Hence, for $v:=v_{1}-k_{1}=v_{2}+k_{2}$, we have $\left(\phi_{1}\left(v_{1}\right), \phi_{2}\left(v_{2}\right)\right)=\left(\phi_{1}(v), \phi_{2}(v)\right)=\eta(v)$.

To end with, let us remark that an appropriate version of this lemma becomes a theorem characterising the exact Maltsev categories among the regular categories [CKP93].

2.2. Distributive lattices. To reformulate coverings in terms of flabby sheaves, we will need a method yielding a presentation of the elements of finitely generated distributive lattices. To this end, we consider first the lattice of upper sets. We call a set $\alpha$ of non-empty subsets of $\{1, \ldots, N\}$ an upper set (or a dual order ideal) if

$$
a \in \alpha \quad \text { and } \quad a \subseteq b \Rightarrow b \in \alpha .
$$

We denote by $\Upsilon_{N} \subseteq 2^{2^{\{1, \ldots, N\}}}$ the set of all non-empty upper sets in the set $2^{\{1, \ldots, N\}}$ of all subsets of $\{1, \ldots, N\}$. The set union and intersection make $\Upsilon_{N}$ a distributive lattice. Furthermore, the upper sets are non-empty open sets of $2^{\{1, \ldots, N\}}$ in the Alexandrov topology. One can prove that $\Upsilon_{N}$ is generated as a distributive lattice by the sets

$$
S_{i}:=\left\{a \subseteq 2^{\{1, \ldots, N\}} \mid i \in a\right\}, \quad i \in\{1, \ldots, N\} .
$$

We want to show that $\Upsilon_{N}$ is isomorphic to the free distributive lattice generated by $N$ elements (see [B-G67], par. III.4). This means verifying that $\Upsilon_{N}$ enjoys the universal property defining the free distributive lattice with $N$-generators. For any lattice $(\Lambda, \vee, \wedge)$ generated by $\lambda_{1}, \ldots, \lambda_{N}$ we can define a map

$$
R^{\left(\lambda_{i}\right)_{i}}: \Upsilon_{N} \ni \alpha \longmapsto \underset{\left\{i_{1}, \ldots, i_{k}\right\} \in \alpha}{\bigvee}\left(\lambda_{i_{1}} \wedge \cdots \wedge \lambda_{i_{k}}\right) \in \Lambda
$$

It is a homomorphism of lattices if, for all $\alpha_{1}, \alpha_{2} \in \Upsilon_{N}$,

$$
\begin{aligned}
& R^{\left(\lambda_{i}\right)_{i}}\left(\alpha_{1} \cap \alpha_{2}\right)=R^{\left(\lambda_{i}\right)_{i}}\left(\alpha_{1}\right) \wedge R^{\left(\lambda_{i}\right)_{i}}\left(\alpha_{2}\right), \\
& R^{\left(\lambda_{i}\right)_{i}}\left(\alpha_{1} \cup \alpha_{2}\right)=R^{\left(\lambda_{i}\right)_{i}}\left(\alpha_{1}\right) \vee R^{\left(\lambda_{i}\right)_{i}}\left(\alpha_{2}\right) .
\end{aligned}
$$

The second equality of (6) always holds. To prove the first one, we need to assume that the lattice $\Lambda$ is distributive. First, observe that

$$
\alpha_{1} \cap \alpha_{2}=\left\{a_{1} \cup a_{2} \mid a_{1} \in \alpha_{1}, a_{2} \in \alpha_{2}\right\}
$$

for all $\alpha_{1}, \alpha_{2} \in \Upsilon_{N}$. Indeed, since $a_{1} \subseteq a_{1} \cup a_{2}$ and $a_{2} \subseteq a_{1} \cup a_{2}$, the left-hand side contains the right-hand side. The other inclusion follows from $\alpha_{1} \cap \alpha_{2} \subseteq \alpha_{1}$, $\alpha_{1} \cap \alpha_{2} \subseteq \alpha_{2}$ and $a=a \cup a$. Now, by the distributivity of $\Lambda$, formula (7) implies the first equation of (6). 
Distributive lattices resemble polynomials with no constant term. The constant term corresponds to the empty set of generators. In case of a lattice of ideals in an algebra $P$ (with $\vee=\cap$ and $\wedge=+$ ), it is important to extend $R^{\left(\lambda_{i}\right)_{i}}$ to a homomorphism of extended lattices $\Upsilon_{N} \cup\{\emptyset\} \rightarrow \Lambda \cup\{P\}$ by declaring that its value on $\emptyset$ is $P$. We will abuse the notation by denoting the thus extended homomorphism with the same symbol. Taking into account the standard convention that the intersection of the empty family of subsets is the whole space, we obtain this way a natural extension of (5). In the same spirit, we need to think of the zero algebra as the unital algebra of all continuous functions on the empty set. The unique possible map from a unital algebra into the zero algebra is then understood as a homomorphism of unital algebras.

Next, let $P$ be an algebra, and let $\left(I_{i}\right)_{i \in\{1, \ldots, N\}}$ be an ordered family of ideals of $P$ intersecting to zero and generating a distributive lattice $\Xi_{N}$ with + and $\cap$ as meet and join operations, respectively. The pairs $\left(P,\left(I_{i}\right)_{i \in\{1, \ldots, N\}}\right)$ form a category $\boldsymbol{I}_{N}$ of distributive lattices of ideals. A morphism between $\left(P,\left(I_{i}\right)_{i \in\{1, \ldots, N\}}\right)$ and $\left(Q,\left(J_{i}\right)_{i \in\{1, \ldots, N\}}\right)$ is, by definition, an algebra map $f: P \rightarrow Q$ such that $f\left(I_{i}\right) \subseteq J_{i}$, for all $i \in\{1, \ldots, N\}$. On the other hand, we consider the category $\boldsymbol{F}_{N}$ of flabby sheaves of algebras over the space $\mathbb{S}_{N}:=2^{\{1, \ldots, N\}} \backslash\{\emptyset\}$ with the Alexandrov topology (open sets are upper sets). Since every sheaf is a functor, we define as morphisms between sheaves natural transformations of functors.

Proposition 2.2. Let $\boldsymbol{I}_{N}$ be the category of distributive lattices of ideals generated by $N$ elements, and $\boldsymbol{F}_{N}$ be the category of flabby sheaves of algebras over $\$_{N}$. Then the assignments

$$
\begin{gathered}
\boldsymbol{I}_{N} \ni\left(P,\left(I_{i}\right)_{i \in\{1, \ldots, N\}}\right) \stackrel{F}{\mapsto}\left\{\mathcal{P}: U \mapsto P / R^{\left(I_{i}\right)_{i}}(U)\right\}_{U} \in \boldsymbol{F}_{N}, \\
\boldsymbol{F}_{N} \ni \mathcal{P} \stackrel{G}{\mapsto}\left(\mathcal{P}\left(\mathbb{S}_{N}\right),\left(\operatorname{ker}\left(\mathcal{P}\left(\mathbb{S}_{N}\right) \rightarrow \mathcal{P}\left(S_{i}\right)\right)\right)_{i}\right) \in \boldsymbol{I}_{N},
\end{gathered}
$$

are functors establishing an equivalence of categories.

Proof. Assume that we are given an object $\left(P,\left(I_{i}\right)_{i \in\{1, \ldots, N\}}\right) \in \boldsymbol{I}_{N}$. We want to show that (8) assigns to it a flabby sheaf. For brevity, we put $R^{\left(I_{i}\right)_{i}}=R$. Let $U, U^{\prime}$ be open subsets of $\Phi_{N}$ such that $U \subseteq U^{\prime}$. It follows from (6) that $R\left(U^{\prime}\right) \subseteq R(U)$. Therefore, one can define the restriction maps

$$
\pi_{U^{\prime}, U}: \mathcal{P}\left(U^{\prime}\right) \rightarrow \mathcal{P}(U), \quad p+R\left(U^{\prime}\right) \mapsto p+R(U),
$$

so that $\mathcal{P}$ is a presheaf. Next, let $U$ be an open subset of $\$_{N}$ and let $\left\{U_{k}\right\}_{k}$ be an open covering of $U$ (i.e., $\left.\bigcup_{k} U_{k}=U\right)$. Consider elements $p_{k} \in \mathcal{P}\left(U_{k}\right)$ such that $\pi_{U_{k}, U_{k} \cap U_{l}}\left(p_{k}\right)=\pi_{U_{l}, U_{k} \cap U_{l}}\left(p_{l}\right)$, for all $k, l$. By the distributivity of the lattice $\Xi_{N}$ and the generalised Chinese Remainder Theorem (e.g., see [SS58], Theorem 18 on p. 280), there exists an element $p_{U} \in \mathcal{P}(U)$ such that $\pi_{U, U_{k}}\left(p_{U}\right)=p_{k}$ for all $k$. To prove the uniqueness of $p_{U}=: p+R(U)$, suppose that $\pi_{U, U_{k}}\left(p_{U}\right):=p+R\left(U_{k}\right)=$ 0 for all $k$. This means that $p \in \bigcap_{k} R\left(U_{k}\right)$. On the other hand, it follows from (6) 
that $R\left(U \cup U^{\prime}\right)=R(U) \cap R\left(U^{\prime}\right)$, for any open subsets $U, U^{\prime}$ of $\$_{N}$. Consequently, $p \in R\left(\bigcup_{k} U_{k}\right)=R(U)$, that is $p_{U}=0$. This establishes the desired uniqueness. Hence $\mathcal{P}$ is a flabby sheaf, and the assignment $F$ is well defined.

Now suppose that we are given a flabby sheaf $\mathcal{P}$ of algebras over $\mathbb{S}_{N}$. Let $\pi_{V, U}: \mathcal{P}(V) \rightarrow \mathcal{P}(U)$ denote the restriction map for any open subsets $U$ and $V$ such that $U \subseteq V$. For brevity, we write $\pi_{U}$ instead of $\pi_{V, U}$ if $V=\mathbb{S}_{N}$. By the flabbiness of $\mathcal{P}$, the morphisms $\pi_{S_{i}}$ (see (4)) are surjective. The property $\bigcap_{i=1}^{N} \operatorname{ker} \pi_{S_{i}}=$ $\{0\}$ follows from the sheaf condition. Furthermore, as lattices of sets are always distributive, to prove the distributivity of the lattice $\Xi_{N}$ it is enough to show that the assignment $U \mapsto \operatorname{ker} \pi_{U}$ defines a surjective morphism from $\Upsilon_{N}$ onto $\Xi_{N}$ transforming the union and intersection of open subsets to the intersection and sum of ideals respectively. To show this, let $U^{\prime}$ and $U^{\prime \prime}$ be open subsets of $\$_{N}$. Since $\mathcal{P}$ is a sheaf, we know that $\mathcal{P}\left(U^{\prime} \cup U^{\prime \prime}\right)$ is the fibre product of $\mathcal{P}\left(U^{\prime}\right)$ and $\mathcal{P}\left(U^{\prime \prime}\right)$. Hence (3) implies that

$$
\operatorname{ker} \pi_{U^{\prime} \cup U^{\prime \prime}}=\operatorname{ker} \pi_{U^{\prime}} \cap \operatorname{ker} \pi_{U^{\prime \prime}},
$$

as needed. Similarly, since the sheaf $\mathcal{P}$ is flabby, Lemma 2.1 implies that

$$
\operatorname{ker} \pi_{U^{\prime} \cap U^{\prime \prime}}=\operatorname{ker} \pi_{U^{\prime}}+\operatorname{ker} \pi_{U^{\prime \prime}} \text {. }
$$

Thus we have shown that (9) assigns to flabby sheaves objects of $\boldsymbol{I}_{N}$ so that the assignment $G$ is well defined.

The functoriality of $G$ is immediate. Indeed, let $\Phi: \mathcal{P} \rightarrow \mathcal{Q}$ be a natural transformation of flabby sheaves of algebras over $\$_{N}$. Denote by $\eta$ the restriction morphisms of $\mathcal{Q}$. Then, since $\eta_{S_{i}} \circ \Phi_{\Phi_{N}}=\Phi_{S_{i}} \circ \pi_{S_{i}}$, we have $\Phi_{\Phi_{N}}\left(\operatorname{ker} \pi_{S_{i}}\right) \subseteq \operatorname{ker} \eta_{S_{i}}$. To prove that $F$ is a functor, consider an algebra homomorphism $f: P \rightarrow Q$ such that $f\left(I_{i}\right) \subseteq J_{i}$ for all $i$. (It is a morphism in $\boldsymbol{I}_{N}$.) For any open subset $U$, we obtain an analogous inclusion $f\left(R^{\left(I_{i}\right)_{i}}(U)\right) \subseteq R^{\left(J_{i}\right)_{i}}(U)$. Hence we have algebra homomorphisms

$$
P / R^{\left(I_{i}\right)_{i}}(U) \rightarrow Q / R^{\left(J_{i}\right)_{i}}(U), \quad p+R^{\left(I_{i}\right)_{i}}(U) \mapsto f(p)+R^{\left(J_{i}\right)_{i}}(U),
$$

defining the desired morphism of sheaves.

It is clear that $G \circ F$ is a functor naturally isomorphic to the identity functor on the category $\boldsymbol{I}_{N}$ because $R^{\left(I_{i}\right)_{i}}\left(S_{j}\right)=I_{j}$ for any index $j$. On the other hand, consider an object $\mathcal{P}$ in the category $\boldsymbol{F}_{N}$ of flabby sheaves. Combining (10) and (11) with the fact that $R^{\left(I_{i}\right)_{i}}$ is a morphism of lattices, we can conclude that there is always a natural isomorphism $\mathcal{P}(U) \cong \mathcal{P}\left(\Phi_{N}\right) / R^{\left(I_{i}\right)_{i}}(U)$. Hence $F \circ G$ is naturally isomorphic to the identity functor on $\boldsymbol{F}_{N}$.

2.3. Principal comodule algebras. Let $(H, \Delta, \varepsilon, S)$ be a Hopf algebra with bijective antipode. A right $H$-comodule algebra $P$ is a unital associative algebra equipped with an $H$-coaction $\Delta_{P}: P \rightarrow P \otimes H$ that is an algebra map. For a comodule algebra $P$, we call

$$
P^{\mathrm{co} H}:=\left\{p \in P \mid \Delta_{P}(p)=p \otimes 1\right\}
$$


the subalgebra of coaction-invariant elements in $P$. The assumed existence of the inverse of the antipode allows us to define a left coaction ${ }_{P} \Delta: P \rightarrow H \otimes P$ by the formula $p \mapsto S^{-1}\left(p_{(1)}\right) \otimes p_{(0)}$. This makes $P$ a left $H$-comodule, and a left $H^{\text {op }}$-comodule algebra.

Definition 2.3. Let $P$ be a right comodule algebra over a Hopf algebra $H$ with bijective antipode, and let $B:=P^{\mathrm{co} H}$ be the coaction-invariant subalgebra. The comodule algebra $P$ is called principal if the following conditions are satisfied:

(1) the coaction of $H$ is Galois, that is, the map (called the canonical map)

$$
\text { can }: P \otimes_{B} P \rightarrow P \otimes H, \quad p \otimes q \mapsto p q_{(0)} \otimes q_{(1)},
$$

is bijective,

(2) the comodule algebra $P$ is right $H$-equivariantly projective as a left $B$-module, i.e., there exists a right $H$-colinear and left $B$-linear splitting of the multiplication map $B \otimes P \rightarrow P$.

This splitting can always be chosen to be unital [BH04]. Also, in this setting, one can show that the $H$-equivariant projectivity of $P$ over $B$ is equivalent to the faithful flatness of $P$ over $B$ [SS05], [SS]. If $P$ is a principal comodule algebra, then the extension of algebras $B \subseteq P$ is a special case of principal extensions defined in [BH04].

A cleft Hopf-Galois extension (e.g., a smash product $B$ \# $H$ of an $H$-module algebra $B$ by $H$ ) is always principal. Indeed, by [BM89], p. 42, cleft Hopf-Galois extensions always enjoy the normal basis property, and the latter can be viewed as equivariant freeness, a special case of equivariant projectivity. For more details and an introduction to Hopf-Galois theory see, e.g., [M-S93], [S-HJ94].

2.4. Strong connections. The inverse of the canonical map defines a monomorphism $H \rightarrow P \otimes_{B} P, h \mapsto$ can $^{-1}(1 \otimes h)$, called the translation map. It turns out that lifts of this map to $P \otimes P$ that are both right and left $H$-colinear yield an equivalent approach to principality [BH04]:

Definition 2.4. Let $H$ be a Hopf algebra with bijective antipode, and $\widetilde{c a n}: P \otimes P \rightarrow$ $P \otimes H$ be the lift of can to $P \otimes P$. Then a strong connection (cf. [H-PM96], [DGH01]) on a right $H$-comodule algebra $P$ is a unital linear map $\ell: H \rightarrow P \otimes P$ satisfying

$\left(\operatorname{id}_{P} \otimes \Delta_{P}\right) \circ \ell=\left(\ell \otimes \operatorname{id}_{H}\right) \circ \Delta, \quad\left(P \Delta \otimes \operatorname{id}_{P}\right) \circ \ell=\left(\operatorname{id}_{H} \otimes \ell\right) \circ \Delta, \quad \widetilde{c a n} \circ \ell=1 \otimes \mathrm{id}$.

The last property of the strong connection (splitting of $\widetilde{\text { can }}$ ) gives rise to the 
commutative diagram

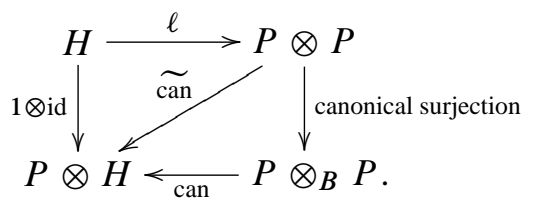

Using the Heyneman-Sweedler-type notation $h \mapsto \ell(h)^{\langle 1\rangle} \otimes \ell(h)^{\langle 2\rangle}$ (summation suppressed), we can write the bicolinearity and splitting property of a strong connection as follows:

$$
\begin{aligned}
\ell(h)^{\langle 1\rangle} \otimes \ell(h)_{(0)}^{\langle 2\rangle} \otimes \ell(h)_{(1)}^{\langle 2\rangle} & =\ell\left(h_{(1)}\right)^{\langle 1\rangle} \otimes \ell\left(h_{(1)}\right)^{\langle 2\rangle} \otimes h_{(2)}, \\
\ell(h)^{\langle 1\rangle}{ }_{(0)} \otimes \ell(h)^{\langle 1\rangle}{ }_{(1)} \otimes \ell(h)^{\langle 2\rangle} & =\ell\left(h_{(2)}\right)^{\langle 1\rangle} \otimes S\left(h_{(1)}\right) \otimes \ell\left(h_{(2)}\right)^{\langle 2\rangle}, \\
\ell(h)^{\langle 1\rangle} \ell(h)_{(0)}^{\langle 2\rangle}{ }_{(1)} \otimes \ell(h)^{\langle 2\rangle} & =1 \otimes h .
\end{aligned}
$$

Applying id $\otimes \varepsilon$ to the last equation yields a very useful formula:

$$
\ell(h)^{\langle 1\rangle} \ell(h)^{\langle 2\rangle}=\varepsilon(h) .
$$

One can prove that an $H$-comodule algebra $P$ is principal if and only if it admits a strong connection [BH04], [BB05]. Given a strong connection $\ell$, one can show that the formula

$$
P \otimes H \rightarrow P \underset{B}{\otimes} P, \quad p \otimes h \mapsto p \ell(h)^{\langle 1\rangle} \underset{B}{\otimes} \ell(h)^{\langle 2\rangle},
$$

defines the inverse of the canonical map can, so that the coaction of $H$ is Galois. Next, one can also show that

$$
s: P \ni p \mapsto p_{(0)} \ell\left(p_{(1)}\right)^{\langle 1\rangle} \otimes \ell\left(p_{(1)}\right)^{\langle 2\rangle} \in B \otimes P
$$

is a splitting whose existence proves the equivariant projectivity. Much as above, one argues that the formula

$$
s^{\prime}: P \ni p \mapsto \ell\left(S^{-1}\left(p_{(1)}\right)\right)^{\langle 1\rangle} \otimes \ell\left(S^{-1}\left(p_{(1)}\right)\right)^{\langle 2\rangle} p_{(0)} \in P \otimes B
$$

provides a left $H$-colinear and right $B$-linear splitting of the multiplication map $P \otimes B \rightarrow P$.

2.5. Actions of compact quantum groups. Let $\bar{H}$ be the $\mathrm{C}^{*}$-algebra of a compact quantum group in the sense of Woronowicz [W-SL87], [W-SL98] and $H$ its dense Hopf $*$-subalgebra spanned by the matrix coefficients of the irreducible unitary corepresentations. Let $\bar{P}$ be a unital $\mathrm{C}^{*}$-algebra and let $\delta: \bar{P} \rightarrow \bar{P} \otimes_{\min } \bar{H}$ be an injective $\mathrm{C}^{*}$-algebraic right coaction of $\bar{H}$ on $\bar{P}$. (See [BS93], Definition 0.2, for a general definition and [B-FP95], Definition 1, for the special case of compact 
quantum groups.) Then the subalgebra $P \subseteq \bar{P}$ of elements for which the coaction lands in $\bar{P} \otimes H$ (algebraic tensor product),

$$
P:=\{p \in \bar{P} \mid \delta(p) \in \bar{P} \otimes H\},
$$

is an $H$-comodule algebra. It follows from the results of [B-FP95] and [P-P95] that $P$ is dense in $\bar{P}$. We call $P$ the Peter-Weyl comodule algebra associated to the $\mathrm{C}^{*}$-algebraic coaction $\delta$. It is straightforward to verify that the operation $\bar{P} \mapsto P$ is a functor commuting with taking fibre products. Note also that $\bar{P}^{\operatorname{co} \bar{H}}=P^{\mathrm{co} H}$.

\section{Piecewise principality}

To show the piecewise nature of principality, we begin by proving lemmas concerning quotients and fibre products of principal comodule algebras.

Lemma 3.1. Let $\pi: P \rightarrow Q$ be a surjection of right $H$-comodule algebras (bijective antipode assumed). If $P$ is principal, then:

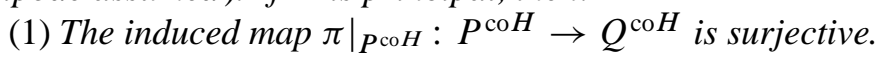

(2) There exists a unital $H$-colinear splitting of $\pi$.

Proof. It follows from the colinearity of $\pi$ that $\pi\left(P^{\mathrm{co} H}\right) \subseteq Q^{\mathrm{co} H}$. To prove the converse inclusion, we take advantage of the left $P^{\mathrm{co} H}$-linear retraction of the inclusion $P^{\mathrm{co} H} \subseteq P$ that was used to prove [BH04], Theorem 2.5 (3):

$$
\sigma: P \rightarrow P^{\mathrm{co} H}, \quad \sigma(p):=p_{(0)} \ell\left(p_{(1)}\right)^{\langle 1\rangle} \varphi\left(\ell\left(p_{(1)}\right)^{\langle 2\rangle}\right) .
$$

Here $\ell$ is a strong connection on $P$ and $\varphi$ is any unital linear functional on $P$. If $\pi(p) \in Q^{\mathrm{co} H}$, then $\sigma(p)$ is a desired element of $P^{\mathrm{co} H}$ that is mapped by $\pi$ to $\pi(p)$. Indeed, since $\pi\left(p_{(0)}\right) \otimes p_{(1)}=\pi(p) \otimes 1$, using the unitality of $\ell, \pi$ and $\varphi$, we compute

$$
\pi(\sigma(p))=\pi\left(p_{(0)}\right) \pi\left(\ell\left(p_{(1)}\right)^{\langle 1\rangle}\right) \varphi\left(\ell\left(p_{(1)}\right)^{\langle 2\rangle}\right)=\pi(p) .
$$

To prove the second assertion, let us choose any $k$-linear unital splitting of $\left.\pi\right|_{P \mathrm{co} H}$ and denote it by $\alpha^{\mathrm{co} H}$. One can readily verify that the formula

$$
\alpha(q):=\alpha^{\mathrm{co} H}\left(q_{(0)} \pi\left(\ell\left(q_{(1)}\right){ }^{\langle 1\rangle}\right)\right) \ell\left(q_{(1)}\right)^{\langle 2\rangle}
$$

defines a unital colinear splitting of $\pi$. (Note also that $\alpha^{\mathrm{co} H}=\left.\alpha\right|_{Q^{\mathrm{co} H} \text {.) }}$

Lemma 3.2. Let $P$ be a fibre product in the category of right $H$-comodule algebras:

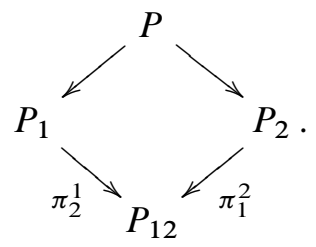


If $P_{1}$ and $P_{2}$ are principal and $\pi_{2}^{1}$ and $\pi_{1}^{2}$ are surjective, then $P$ is a principal comodule algebra.

Proof. Given strong connections $\ell_{1}$ and $\ell_{2}$ on $P_{1}$ and $P_{2}$ respectively, we want to construct a strong connection on $P$. A first approximation for such a strong connection is as follows:

$$
\lambda: H \rightarrow P \otimes P, \quad \lambda(h):=\left(\ell_{1}(h)^{\langle 1\rangle}, f_{2}^{1}\left(\ell_{1}(h)^{\langle 1\rangle}\right)\right) \otimes\left(\ell_{1}(h)^{\langle 2\rangle}, f_{2}^{1}\left(\ell_{1}(h)^{\langle 2\rangle}\right)\right) .
$$

Here $f_{2}^{1}:=\sigma_{2} \circ \pi_{2}^{1}$ and $\sigma_{2}$ is a unital colinear splitting of $\pi_{1}^{2}$, which exists by Lemma 3.1 (2). (Note that our Heyneman-Sweedler-type summation convention for strong connections $x^{\langle 1\rangle} \otimes x^{\langle 2\rangle}=\sum_{i} x_{i} \otimes y_{i}$ is extended to the direct products by the formula $\left(x^{\langle 1\rangle}, x^{\langle 1\rangle}\right) \otimes\left(x^{\langle 2\rangle}, x^{\langle 2\rangle}\right):=\sum_{i}\left(x_{i}, x_{i}\right) \otimes\left(y_{i}, y_{i}\right)$.) The map $\lambda$ is unital and bicolinear, but it does not split the lifted canonical map:

$$
\begin{aligned}
(1,1) & \otimes h-\widetilde{\operatorname{can}}(\lambda(h)) \\
& =(0,1) \otimes h-\left(0, f_{2}^{1}\left(\ell_{1}\left(h_{(1)}\right)^{\langle 1\rangle}\right) f_{2}^{1}\left(\ell_{1}\left(h_{(1)}\right)^{\langle 2\rangle}\right)\right) \otimes h_{(2)} \in P_{2} \otimes H .
\end{aligned}
$$

Now, let $\widetilde{\mathrm{can}_{2}}$ be the lifted canonical map on $P_{2} \otimes P_{2}$. Applying the splitting of $\widetilde{\text { aan }_{2}}$ given by $\ell_{2}$ (cf. (13)) to the right-hand side of the above equation, gives a correction term for $\lambda$ :

$$
\begin{aligned}
T(h) & :=\ell_{2}(h)-f_{2}^{1}\left(\ell_{1}\left(h_{(1)}\right)^{\langle 1\rangle}\right) f_{2}^{1}\left(\ell_{1}\left(h_{(1)}\right)^{\langle 2\rangle}\right) \ell_{2}\left(h_{(2)}\right)^{\langle 1\rangle} \otimes \ell_{2}\left(h_{(2)}\right)^{\langle 2\rangle} \\
& =\left(\varepsilon\left(h_{(1)}\right)-f_{2}^{1}\left(\ell_{1}\left(h_{(1)}\right)^{\langle 1\rangle}\right) f_{2}^{1}\left(\ell_{1}\left(h_{(1)}\right)^{\langle 2\rangle}\right)\right) \ell_{2}\left(h_{(2)}\right)^{\langle 1\rangle} \otimes \ell_{2}\left(h_{(2)}\right)^{\langle 2\rangle} .
\end{aligned}
$$

This defines a bicolinear map into $P_{2} \otimes P_{2}$ which annihilates 1 . Considering $\lambda$ as a map into $\left(P_{1} \oplus P_{2}\right)^{\otimes 2}$, we can add these two maps. The map $\lambda+T$ is still unital, bicolinear and splits the lifted canonical map on $\left(P_{1} \oplus P_{2}\right)^{\otimes 2}$. Remembering (1) and (12), it is clear from the formula for $T$ that to make it take values in $P \otimes P$ we only need to add the term

$$
T^{\prime}(h):=\left(\varepsilon\left(h_{(1)}\right)-f_{2}^{1}\left(\ell_{1}\left(h_{(1)}\right)^{\langle 1\rangle}\right) f_{2}^{1}\left(\ell_{1}\left(h_{(1)}\right)^{\langle 2\rangle}\right)\right) \ell_{2}\left(h_{(2)}\right)^{\langle 1\rangle} \otimes f_{1}^{2}\left(\ell_{2}\left(h_{(2)}\right)^{\langle 2\rangle}\right) .
$$

Much as above, here $f_{1}^{2}:=\sigma_{1} \circ \pi_{1}^{2}$ and $\sigma_{1}$ is a unital colinear splitting of $\pi_{2}^{1}$, which exists by Lemma 3.1 (2). The formula (16) defines a bicolinear map into $P_{2} \otimes P_{1}$ which annihilates 1 . Since the lifted canonical map on $\left(P_{1} \oplus P_{2}\right)^{\otimes 2}$ vanishes on $P_{2} \otimes P_{1}$, the sum $\lambda+T+T^{\prime}$ splits the lifted canonical map, takes values in $P \otimes P$ and is unital and bicolinear. Thus it is, as desired, a strong connection on $P$.

Let us now consider a family $\pi_{i}: P \rightarrow P_{i}, i \in\{1, \ldots, N\}$, of surjections of right $H$-comodule algebras with $\bigcap_{i=1}^{N} \operatorname{ker} \pi_{i}=0$. Denote $J_{i}:=\operatorname{ker} \pi_{i}$. By Lemma 2.1 and formula (3), for any $k=1, \ldots, N-1$ there is a fibre-product diagram of right 
$H$-comodule algebras

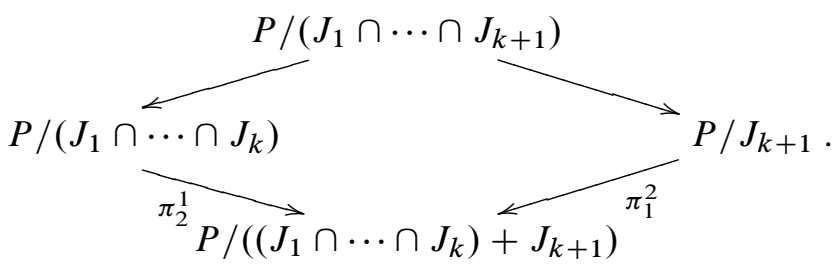

Assume that for all $i$ the comodule algebras $P_{i} \cong P / J_{i}$ are principal. Then Lemma 3.2 implies by an obvious induction that $P /\left(J_{1} \cap \cdots \cap J_{k}\right)$ is principal for all $k=1, \ldots, N$. In particular $(k=N), P$ is principal. On the other hand, if $P$ is principal then all the $P_{i}$ 's are principal. Indeed, if $\ell: H \rightarrow P \otimes P$ is a strong connection on $P$, then

$$
\left(\pi_{i} \otimes \pi_{i}\right) \circ \ell: H \longrightarrow P_{i} \otimes P_{i}
$$

is a strong connection on $P_{i}$. Thus we have proved the following:

Theorem 3.3. Let $\pi_{i}: P \rightarrow P_{i}, i \in\{1, \ldots, N\}$, be surjections of right $H$-comodule algebras such that $\bigcap_{i=1}^{N} \operatorname{ker} \pi_{i}=0$. Then $P$ is principal if and only if all the $P_{i}$ 's are principal.

Our next step is a statement about a relation between the ideals of a principal comodule algebra $P$ that are also subcomodules, and ideals in the subalgebra $B$ of coaction-invariant elements. Both sets are obviously lattices with respect to the operations + and $\cap$.

Proposition 3.4. Let $P$ be a principal right $H$-comodule algebra and $B:=P^{\mathrm{co} H}$ the coaction-invariant subalgebra. Denote by $\Xi_{B}$ the lattice of all ideals in $B$ and by $\Xi_{P}$ the lattice of all ideals in $P$ that are simultaneously subcomodules. Then the map

$$
\mathscr{L}: \Xi_{P} \rightarrow \Xi_{B}, \quad \mathscr{L}(J):=J \cap B,
$$

is a monomorphism of lattices.

Proof. The only non-trivial step in proving that $\mathscr{L}$ is a homomorphism of lattices is establishing the inclusion $(B \cap J)+\left(B \cap J^{\prime}\right) \supseteq B \cap\left(J+J^{\prime}\right)$. To this end, we proceed along the lines of the proof of Lemma 3.1(1). Since $J$ is a comodule and an ideal, from the formula (14) we obtain

$$
p \in J \Longrightarrow s(p)=p_{(0)} \ell\left(p_{(1)}\right)^{\langle 1\rangle} \otimes \ell\left(p_{(1)}\right)^{\langle 2\rangle} \in(J \cap B) \otimes P .
$$

Now, let $p \in J, q \in J^{\prime}, p+q \in B$. Then (17) implies that

$$
(p+q) \otimes 1=s(p)+s(q) \in(B \cap J) \otimes P+\left(B \cap J^{\prime}\right) \otimes P .
$$


Applying any unital linear functional $P \rightarrow k$ to the second tensor component implies that $p+q \in(B \cap J)+\left(B \cap J^{\prime}\right)$. Finally, since $s$ is a splitting of the multiplication map, it follows from (17) that $J=(J \cap B) P$. This, in turn, proves the injectivity of $\mathscr{L}$.

Note that, much as the formula (14) implies (17), the formula (15) implies

$$
p \in J \Longrightarrow s^{\prime}(p)=\ell\left(S^{-1}\left(p_{(1)}\right)\right)^{\langle 1\rangle} \otimes \ell\left(S^{-1}\left(p_{(1)}\right)\right)^{\langle 2\rangle} p_{(0)} \in P \otimes(J \cap B) .
$$

Therefore, $J=P(J \cap B)$ since $s^{\prime}$ is a splitting of the right multiplication map. Combining this with the above discussed left-sided version proves that $J$ is a twosided ideal:

$$
P(J \cap B)=J=(J \cap B) P .
$$

Remark 3.5. The homomorphism $\mathscr{L}$ is not surjective in general. A counterexample is given by a smash product (trivial principal comodule algebra) of the Laurent polynomials $B=k\left[u, u^{-1}\right]$ with the Hopf algebra $H=k\left[v, v^{-1}\right]$ of Laurent polynomials $(\Delta(v)=v \otimes v)$. The action is defined by $v \triangleright u=q u, q \in k \backslash\{0,1\}$. Viewing $u$ and $v$ as generators of $P$, it is clear that $v u=q u v$. It is straightforward to verify that if $I$ is the two-sided ideal in $B$ generated by $u-1$, then the right ideal $I P$ is not a two-sided ideal of $P$. Hence the map $\mathscr{L}$ cannot be surjective by (18).

In [CM00], families $\pi_{i}: P \rightarrow P_{i}$ of algebra homomorphisms as in Theorem 3.3 were called coverings. However, it was explained therein (see Proposition 2) that such coverings are well-behaved when the kernels ker $\pi_{i}$ generate a distributive lattice of ideals. Therefore, we adopt in the present paper the following terminology.

Definition 3.6. A finite family $\left\{\pi_{i}: P \rightarrow P_{i}\right\}_{i \in\{1, \ldots, N\}}$ of surjective algebra homomorphisms is called a weak covering if $\bigcap_{i=1, \ldots, N}$ ker $\pi_{i}=\{0\}$. Denote by $\Xi_{N}$ the lattice of ideals generated by $I_{i}:=\operatorname{ker} \pi_{i}, i=1, \ldots, N$, with $\cap$ and + as the join and meet operations respectively. A weak covering is called a covering if the lattice $\Xi_{N}$ is distributive. Finally, an ordered family $\left(\pi_{i}: P \rightarrow P_{i}\right)_{i \in\{1, \ldots, N\}}$ is called an ordered covering if the set $\left\{\pi_{i}: P \rightarrow P_{i}\right\}_{i \in\{1, \ldots, N\}}$ is a covering.

The above definition can obviously be extended to the case when the $\pi_{i}$ 's are algebra and $H$-comodule morphisms. Then the ker $\pi_{i}$ 's are ideals and $H$-subcomodules. Note also that the reason for distinguishing between coverings and ordered coverings comes from the categorical considerations of the following section. To compare coverings with appropriate sheaves of algebras, it is more convenient to use the category of ordered coverings.

The next claim is concerned with the distributivity condition from Definition 3.6 for coverings of principal comodule algebras. It follows from Proposition 3.4 and Lemma 3.1 (1). 
Corollary 3.7. Let $\left\{\pi_{i}: P \rightarrow P_{i}\right\}_{i \in\{1, \ldots, N\}}$ be a family of surjective homomorphisms of right $H$-comodule algebras. Assume that $P$ is principal. Then $\left\{\pi_{i}: P \rightarrow P_{i}\right\}_{i}$ is

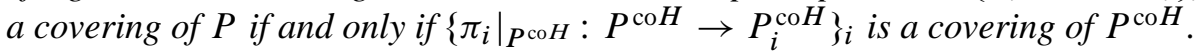

The above corollary is particularly helpful when $P^{\mathrm{co} H}$ is a $\mathrm{C}^{*}$-algebra because lattices of closed ideals in a $\mathrm{C}^{*}$-algebra are always distributive (due to the property $I \cap J=$ $I J)$.

We are now ready to propose a noncommutative-geometric replacement for the concept of local triviality of principal bundles. Recall that the subsets that admit a natural translation into the language of $\mathrm{C}^{*}$-algebras are the closed (not the open) subsets of a compact Hausdorff space. Therefore, we use finite closed rather than open coverings to trivialise bundles. As is explained in [BHMS], Example 1.24, there is a difference between these two approaches. We reserve the term "locally trivial" for bundles trivialisable over an open cover, and call bundles trivialisable over a finite closed cover "piecewise trivial". It is the latter (slightly more general) property that we generalise to the noncommutative setting.

Definition 3.8. An $H$-comodule algebra $P$ is called piecewise principal (respectively, trivial) if there exists a weak covering $\left\{\pi_{i}: P \rightarrow P_{i}\right\}_{i \in\{1, \ldots, N\}}$ by $H$-colinear maps such that:

(1) The restrictions $\left.\pi_{i}\right|_{P^{\mathrm{co} H}}: P^{\mathrm{co} H} \rightarrow P_{i}^{\mathrm{co} H}$ form a covering.

(2) The $P_{i}$ 's are principal (respectively, the $P_{i}$ 's are smash products, i.e., for all $i$, $P_{i} \cong P_{i}^{\operatorname{co} H} \#_{i} H$ as $H$-comodule algebras).

While not every compact principal bundle is piecewise (or locally) trivial ([BHMS], Example 1.22), every piecewise principal compact $G$-space (i.e., covered by finitely many compact principal $G$-bundles) is clearly a compact principal $G$-bundle. The second statement becomes non-trivial when we replace compact $G$-spaces by comodule algebras and follows from Theorem 3.3. Furthermore, as an immediate consequence of Theorem 3.3 and Corollary 3.7, we obtain:

Corollary 3.9. Let $H$ be a Hopf algebra with bijective antipode and $P$ be an $H$ comodule algebra that is piecewise principal with respect to $\left\{\pi_{i}: P \rightarrow P_{i}\right\}_{i}$. Then $P$ is principal and $\left\{\pi_{i}: P \rightarrow P_{i}\right\}_{i}$ is a covering of $P$.

Finally, let us consider the relationship between piecewise triviality and a similar concept referred to as "local triviality" in [P-MJ94]. Therein, sheaves $\mathcal{P}$ of comodule algebras were viewed as quantum analogues of principal bundles. They were called locally trivial provided that the space $X$ on which $\mathcal{P}$ is defined admits an open covering $\left\{U_{i}\right\}_{i}$ such that all $\mathcal{P}\left(U_{i}\right)$ 's are smash products. If we assume such a sheaf to be flabby (that is, for all open subsets $V, U, V \subseteq U$, the restriction maps $\pi_{U, V}: \mathcal{P}(U) \rightarrow \mathcal{P}(V)$ are surjective), then we can use Theorem 3.3 to deduce the principality of all $\mathcal{P}(U)$ 's: 
Corollary 3.10. Let $H$ be a Hopf algebra with bijective antipode and $\mathcal{P}$ be a flabby sheaf of $H$-comodule algebras over a topological space $X$. If $\left\{U_{i}\right\}_{i}$ is a finite open covering such that all $\mathcal{P}\left(U_{i}\right)$ 's are principal, then $\mathcal{P}(U)$ is principal for any open subset $U \subseteq X$.

\section{Coverings and flabby sheaves}

This section is a by-product of our earlier considerations. Its goal is to explain the nature of sheaves encoding noncommutative-geometric coverings. In other words, it is purported to give a flabby-sheaf interpretation of distributive lattices of ideals defining coverings of algebras (see Definition 3.6). We will explain that for a flabby sheaf in Corollary 3.10, the underlying topological space plays only a secondary role and can be replaced by a certain space that is universal for all $N$-element coverings. This space is the 2-element field $(N-1)$-projective space

$$
\mathbb{P}^{N-1}(\mathbb{Z} / 2):=\{0,1\}^{N} \backslash\{(0, \ldots, 0)\}
$$

whose topology subbasis is its affine covering, i.e., whose topology is generated by the subsets

$$
A_{i}:=\left\{\left(z_{1}, \ldots, z_{N}\right) \in \mathbb{P}^{N-1}(\mathbb{Z} / 2) \mid z_{i} \neq 0\right\} .
$$

Consider now an arbitrary space $X$ with a finite covering $\left\{U_{1}, \ldots, U_{N}\right\}$. Define on $X$ the topology generated by the $U_{i}$ 's (considered as open sets) and pass to the quotient by the equivalence relation

$$
x \sim y \Longleftrightarrow\left(\forall i: x \in U_{i} \Longleftrightarrow y \in U_{i}\right) .
$$

Obviously, $X / \sim$ depends on the specific features of the covering $\left\{U_{i}\right\}_{i}$. However, for a fixed $N$, it can always be embedded into $\mathbb{P}^{N-1}(\mathbb{Z} / 2)$ :

Proposition 4.1. Let $X=U_{1} \cup \cdots \cup U_{N}$ be any set equipped with the topology generated by the $U_{i}$ 's. Let $p: X \rightarrow X / \sim$ be the quotient map defined by the equivalence relation (20). Then

$$
\xi: X / \sim \rightarrow \mathbb{P}^{N-1}(\mathbb{Z} / 2), \quad p(x) \mapsto\left(z_{1}, \ldots, z_{N}\right), \quad z_{i}=1 \Longleftrightarrow x \in U_{i} \text { for all } i,
$$

is an embedding of topological spaces.

Proof. It is immediate that $\xi$ is well defined and injective. Next, since $p^{-1}\left(\xi^{-1}\left(A_{i}\right)\right)=U_{i}$ is open for all $i$, each $\xi^{-1}\left(A_{i}\right)$ is open in the quotient topology on $X / \sim$. Now the continuity of $\xi$ follows from the fact that the $A_{i}$ 's form a subbasis of the topology of $\mathbb{P}^{N-1}(\mathbb{Z} / 2)$.

The key step is to show that the images of open sets in $X / \sim$ are open in $\xi(X / \sim)$. First note that by the definition of the relation (20),

$$
p^{-1}\left(p\left(U_{i_{1}} \cap \cdots \cap U_{i_{n}}\right)\right)=U_{i_{1}} \cap \cdots \cap U_{i_{n}} .
$$


Therefore, as preimages and images preserve unions, and any open set in $X$ is the union of intersections of $U_{i}$ 's, $p$ is an open map. On the other hand, by the surjectivity of $p$, we have $p\left(p^{-1}(V)\right)=V$ for any subset $V \subseteq X / \sim$. Hence it follows that a set in $X / \sim$ is open if and only if it is an image under $p$ of an open set in $X$. Finally, by the definition of $\xi$,

$$
\xi\left(p\left(U_{i_{1}} \cap \cdots \cap U_{i_{n}}\right)\right)=A_{i_{1}} \cap \cdots \cap A_{i_{n}} \cap \operatorname{im}(\xi),
$$

and the claim follows from the distributivity of $\cap$ with respect to $U$.

Note that the map $\xi$ is a homeomorphism precisely when the $U_{i}$ 's are in a generic position, that is, when all intersections $U_{i_{1}} \cap \cdots \cap U_{i_{k}} \cap\left(X \backslash U_{j_{1}}\right) \cap \cdots \cap\left(X \backslash U_{j_{l}}\right)$ such that $\left\{i_{1}, \ldots, i_{k}\right\} \cap\left\{j_{1}, \ldots, j_{l}\right\}=\emptyset$ are non-empty.

Thus we have shown that if we consider $X$ and the $U_{i}$ 's as in Corollary 3.10, then the composition $\xi \circ p: X \rightarrow \mathbb{P}^{N-1}(\mathbb{Z} / 2)$ is continuous. Hence we can produce flabby sheaves over $\mathbb{P}^{N-1}(\mathbb{Z} / 2)$ by taking the direct images of flabby sheaves over $X$. They will have the same sections globally and on the covering sets. In this sense, they carry an essential part of the data encoded in the original sheaf.

Example 4.2. We want to show that a closed-set version of the affine covering of $\mathbb{P}^{N-1}(\mathbb{C})$ yields $\xi$ which is a homeomorphism. Denote by $\left[x_{1}: \cdots: x_{N}\right]$ the class of $\left(x_{1}, \ldots, x_{N}\right) \in \mathbb{C}^{N}$ in $\mathbb{P}^{N-1}(\mathbb{C})$. Then the affine subsets

$$
V_{i}=\left\{\left[x_{1}: \cdots: x_{N}\right] \in \mathbb{P}^{N-1}(\mathbb{C}) \mid x_{i} \neq 0\right\}, \quad i=1, \ldots, N,
$$

form a covering of $\mathbb{P}^{N-1}(\mathbb{C})$. Next, define the following refinement of this covering:

$$
X_{i}=\left\{\left[x_{1}: \cdots: x_{N}\right] \in \mathbb{P}^{N-1}(\mathbb{C})|| x_{i} \mid=\max \left\{\left|x_{1}\right|, \ldots,\left|x_{N}\right|\right\}\right\}, \quad i=1, \ldots, N .
$$

It is clear that under the homeomorphisms

$$
\Psi_{i}: V_{i} \rightarrow \mathbb{C}^{N-1}, \quad\left[x_{1}: \cdots: x_{N}\right] \mapsto\left(\frac{x_{1}}{x_{i}}, \ldots, \frac{x_{i-1}}{x_{i}}, \frac{x_{i+1}}{x_{i}}, \ldots, \frac{x_{N}}{x_{i}}\right),
$$

defining the manifold structure of $\mathbb{P}^{N-1}(\mathbb{C})$, the sets $X_{i}$ become closed $(N-1)$ cubes:

$$
\Psi_{i}\left(X_{i}\right)=\left\{\left(y_{1}, \ldots, y_{N-1}\right) \in \mathbb{C}^{N-1}|| y_{j} \mid \leq 1 \text { for all } j\right\} .
$$

Therefore, the $X_{i}$ 's form a closed covering of $\mathbb{P}^{N-1}(\mathbb{C})$. Finally, to show that the embedding $\xi$ induced by this covering is surjective, consider an arbitrary element $\left(z_{1}, \ldots, z_{N}\right) \in \mathbb{P}^{N-1}(\mathbb{Z} / 2)$ that is a non-zero sequence of zeros and ones. Then

$$
(\xi \circ p)\left(\left[z_{1}: \cdots: z_{N}\right]\right)=\left(z_{1}, \ldots, z_{N}\right),
$$

as needed. 
We are now ready to demonstrate that flabby sheaves of algebras over $\mathbb{P}^{N-1}(\mathbb{Z} / 2)$ are just a reformulation of the notion of an ordered covering introduced in Definition 3.6. It turns out that the distributivity condition discussed in the previous section is the key property needed to reconcile the results from [BK96], [CM02] with those from [P-MJ94].

To this end, let us call an ordered covering by $N$ surjections an ordered $N$ covering, and let us define a morphism between ordered $N$-coverings $\left(\pi_{i}: P \rightarrow\right.$ $\left.P_{i}\right)_{i}$ and $\left(\eta_{i}: Q \rightarrow Q_{i}\right)_{i}$ as a family of algebra homomorphisms $f: P \rightarrow Q$ and $f_{i}: P_{i} \rightarrow Q_{i}, i \in\{1, \ldots, N\}$, such that $\eta_{i} \circ f=f_{i} \circ \pi_{i}$ for any index $i$. This category is obviously equivalent to the category $\boldsymbol{I}_{N}$ of distributive lattices of ideals (see Proposition 2.2).

On the other hand, to transform a category of sheaves over the topological space $\mathbb{S}_{N}$ (i.e., over $2^{\{1, \ldots, N\}} \backslash\{\emptyset\}$ with the Alexandrov topology) to an equivalent category of sheaves over $\mathbb{P}^{N-1}(\mathbb{Z} / 2)$, all we need is a homeomorphism between these spaces. This is provided by the obvious bijection

$$
\chi: \mathbb{P}^{N-1}(\mathbb{Z} / 2) \ni\left(z_{i}\right)_{i \in\{1, \ldots, N\}} \mapsto\left\{j \in\{1, \ldots, N\} \mid z_{j}=1\right\} \in \mathbb{S}_{N}
$$

mapping the subbasis of the topology of $\mathbb{P}^{N-1}(\mathbb{Z} / 2)$ to the subbasis of the topology of $\$_{N}$, i.e., $\chi\left(A_{i}\right)=S_{i}, i \in\{1, \ldots, N\}$ (see (19) and (4)).

Hence Proposition 2.2 enjoys the following corollary:

Corollary 4.3. Let $\boldsymbol{C}_{N}$ be the category of ordered $N$-coverings of algebras, and $\boldsymbol{P}_{N}$ be the category of flabby sheaves of algebras over $\mathbb{P}^{N-1}(\mathbb{Z} / 2)$. Then the assignments

$$
\begin{gathered}
C_{N} \ni\left(\pi_{i}: P \rightarrow P_{i}\right)_{i} \mapsto\left\{S: Y \mapsto P /\left(R^{\left(I_{i}\right)_{i}} \circ \chi\right)(Y)\right\}_{Y} \in \boldsymbol{P}_{N}, \\
\boldsymbol{P}_{N} \ni S \mapsto\left(S\left(\mathbb{P}^{N-1}(\mathbb{Z} / 2)\right) \rightarrow S\left(A_{i}\right)\right)_{i} \in C_{N},
\end{gathered}
$$

are functors establishing an equivalence of categories.

Remark 4.4. Let $\Gamma_{N}$ and $\Upsilon_{N}$ denote the lattices of non-empty open subsets of $\mathbb{P}^{N-1}(\mathbb{Z} / 2)$ and $\mathbb{S}_{N}$ respectively. For any $N$, the homeomorphism $\chi$ forces the homomorphism of lattices $R^{\left(A_{i}\right)_{i}}: \Upsilon_{N} \rightarrow \Gamma_{N}$ defined by (5) to be bijective (cf. [B-G67], Theorem 9, p. 34). Its inverse is given by the formula $\left(R^{\left(A_{j}\right)_{j}}\right)^{-1}(Y)=\chi(Y)$.

Let us end this section by showing how Corollary 4.3 works in the classical setting. Since the intersection of closed ideals in a $\mathrm{C}^{*}$-algebra equals their product, the lattices of closed ideals in $\mathrm{C}^{*}$-algebras are always distributive. On the other hand, the category of compact Hausdorff spaces is equivalent to the opposite category of unital commutative $\mathrm{C}^{*}$-algebras (see [GN43], Lemma 1, for the original statement). Therefore, remembering that the epimorphisms of commutative unital $\mathrm{C}^{*}$-algebras can be equivalently presented as the pullbacks of embeddings of compact Hausdorff spaces, we immediately obtain: 
Corollary 4.5. The category of compact Hausdorff spaces $X$ with fixed ordered closed covering $\left(C_{i} \subseteq X\right)_{i \in\{1, \ldots, N\}}$ is equivalent to the opposite category of flabby sheaves of commutative unital $C^{*}$-algebras over $\mathbb{P}^{N-1}(\mathbb{Z} / 2)$.

Recall that here the zero algebra is allowed as a unital $\mathrm{C}^{*}$-algebra. It is "the algebra of all continuous complex-valued functions on the empty set". This is needed if the closed sets are not in generic position. The set of unital morphisms from the zero $\mathrm{C}^{*}$-algebra to any non-zero $\mathrm{C}^{*}$-algebra is understood to be empty.

\section{Examples}

To end with, we recall from [DHH], [HMS06a], [BHMS05] and [HMS06b] certain constructions of algebras and show that they are piecewise trivial comodule algebras. This way we indicate possible areas of applications of Section 3.

5.1. A noncommutative join construction. If $G$ is a compact group, then the join $G * G$ becomes a $G$-principal fibre bundle over the unreduced suspension $\Sigma G$ of $G$ (e.g., see [B-GE93], Proposition VII.8.8). For example, one can obtain in this way the Hopf fibrations $S^{7} \rightarrow S^{4}$ and $S^{3} \rightarrow S^{2}$ using $G=\operatorname{SU}(2)$ and $G=\mathrm{U}(1)$ respectively. Recall that $G * G$ is constructed from $[0,1] \times G \times G$ by shrinking to a point one factor $G$ at 0 and the other one at 1 . Alternatively, at one of the endpoints of the unit interval, one can shrink $G \times G$ to the diagonal. The latter picture is generalised in $[\mathrm{DHH}]$ to the noncommutative setting. Here we construct anew a special case of this generalisation from the piecewise-triviality point of view.
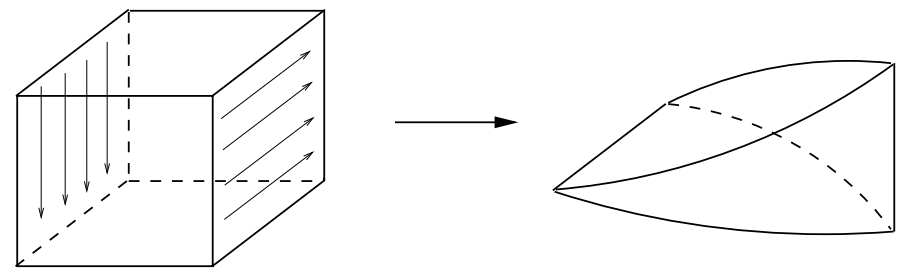

Let $H$ be the Hopf algebra underlying a compact quantum group $\bar{H}$ (see [W-SL87], [W-SL98] or Chapter 11 of [KS97] for details). We define

$$
\begin{aligned}
& P_{1}:=\{f \in C([0,1], \bar{H}) \otimes H \mid f(0) \in \Delta(H)\}, \\
& P_{2}:=\{f \in C([0,1], \bar{H}) \otimes H \mid f(1) \in \mathbb{C} \otimes H\} .
\end{aligned}
$$

They play the roles of two trivial pieces of a principal comodule algebra. Here we identify elements of $C([0,1], \bar{H}) \otimes H$ with functions $[0,1] \rightarrow \bar{H} \otimes H$. The $P_{i}$ 's become $H$-comodule algebras by applying the coproduct of $H$ to $H, \Delta_{P_{i}}=$ 
$\operatorname{id}_{C([0,1], \bar{H})} \otimes \Delta$, and the subalgebras of $H$-coaction invariants can be identified with

$$
\begin{aligned}
& B_{1}:=\{f \in C([0,1], \bar{H}) \mid f(0) \in \mathbb{C}\}, \\
& B_{2}:=\{f \in C([0,1], \bar{H}) \mid f(1) \in \mathbb{C}\} .
\end{aligned}
$$

Clearly, $P_{2}=B_{2} \otimes H$. However, to handle $P_{1}$, we need a cleaving map $j: H \rightarrow P_{1}$, $j(h):=\left(t \mapsto h_{(1)}\right) \otimes h_{(2)}$. It defines an action of $H$ on $B_{1}$ via the formula $h \triangleright p:=j\left(h_{(1)}\right) p j\left(S\left(h_{(2)}\right)\right)$. This action gives a smash product $B_{1} \# H$ isomorphic with $P_{1}$ as a right $H$-comodule algebra. Now one can define $P$ as a gluing of the two pieces along $P_{12}:=\bar{H} \otimes H$, that is, as the pullback

$$
P:=\left\{(p, q) \in P_{1} \oplus P_{2} \mid \pi_{2}^{1}(p)=\pi_{1}^{2}(q)\right\}
$$

of the $P_{i}$ 's along the evaluation maps

$$
\pi_{2}^{1}: P_{1} \rightarrow P_{12}, f \mapsto f(1), \quad \pi_{1}^{2}: P_{2} \rightarrow P_{12}, f \mapsto f(0) .
$$

From Theorem 3.3, we can conclude now that $P$ is principal. In particular, taking $\bar{H}$ to be the $\mathrm{C}^{*}$-algebra of $\mathrm{SU}_{q}(2)$, we can conclude the principality of Pflaum's noncommutative instanton bundle [P-MJ94].

5.2. The Heegaard-type quantum 3 -sphere. Based on the idea of a Heegaard splitting of $S^{3}$ into two solid tori, a noncommutative deformation of $S^{3}$ was proposed in [CM02], [HMS06a], [BHMS05]. On the level of $\mathrm{C}^{*}$-algebras, it can be presented as a fibre product $C\left(S_{p q \theta}^{3}\right)$ of two $C^{*}$-algebraic crossed products $\mathcal{T} \rtimes_{\theta} \mathbb{Z}$ and $\mathcal{T} \rtimes_{-\theta} \mathbb{Z}$ of the Toeplitz algebra $\mathcal{T}$ by $\mathbb{Z}$.

We denote the isometry generating $\mathcal{T}$ in $\mathcal{T} \rtimes_{\theta} \mathbb{Z}$ by $z_{+}$and the isometry generating $\mathcal{T}$ in $\mathcal{T} \rtimes_{-\theta} \mathbb{Z}$ by $z_{-}$. The $\mathbb{Z}$-actions are implemented by unitaries $u_{+}$and $u_{-}$, respectively, in the following way:

$$
1_{\mathbb{Z}} \triangleright_{\theta} z_{+}:=u_{+} z_{+} u_{+}^{-1}:=e^{2 \pi i \theta} z_{+}, \quad 1_{\mathbb{Z}} \triangleright_{-\theta} z_{-}:=u_{-} z_{-} u_{-}^{-1}:=e^{-2 \pi i \theta} z_{-} .
$$

The fibre product is taken over $C\left(S^{1}\right) \rtimes_{\theta} \mathbb{Z}$ with the action $1_{\mathbb{Z} \triangleright} \triangleright_{\theta} Z_{+}:=U_{+} z_{+} U_{+}^{-1}:=$ $e^{2 \pi i \theta} Z_{+}$. Here $Z_{+}$is the generator of $C\left(S^{1}\right)$ and $U_{+}$is the unitary implementing the $\mathbb{Z}$-action. The surjections defining the fibre product are

$$
\begin{aligned}
& \pi_{2}^{1}: \mathcal{T} \rtimes_{\theta} \mathbb{Z} \rightarrow C\left(S^{1}\right) \rtimes_{\theta} \mathbb{Z}, \quad z_{+} \mapsto Z_{+}, \quad u_{+} \mapsto U_{+}, \\
& \pi_{1}^{2}: \mathcal{T} \rtimes_{-\theta} \mathbb{Z} \rightarrow C\left(S^{1}\right) \rtimes_{\theta} \mathbb{Z}, \quad z_{-} \mapsto U_{+}, \quad u_{-} \mapsto Z_{+} .
\end{aligned}
$$

There is a natural $\mathrm{U}(1)$-action on $C\left(S_{p q \theta}^{3}\right)$ corresponding classically to the action in the Hopf fibration, see [HMS06a]. Its restriction to the two crossed products is not the canonical action of $\mathrm{U}(1)$ on the right component of $\mathcal{T} \rtimes_{ \pm \theta} \mathbb{Z}$. However, to obtain the canonical actions one can identify $C\left(S_{p q \theta}^{3}\right)$ with a fibre product of the same crossed products, but formed with respect to the surjections

$$
\begin{aligned}
\hat{\pi}_{2}^{1}: \mathcal{T} \rtimes_{\theta} \mathbb{Z} \rightarrow C\left(S^{1}\right) \rtimes_{\theta} \mathbb{Z}, \quad z_{+} \mapsto Z_{+}, \quad u_{+} \mapsto U_{+}, \\
\hat{\pi}_{1}^{2}: \mathcal{T} \rtimes_{-\theta} \mathbb{Z} \rightarrow C\left(S^{1}\right) \rtimes_{\theta} \mathbb{Z}, \quad z_{-} \mapsto Z_{+}^{-1}, \quad u_{-} \mapsto Z_{+} U_{+} .
\end{aligned}
$$


The identification is given by

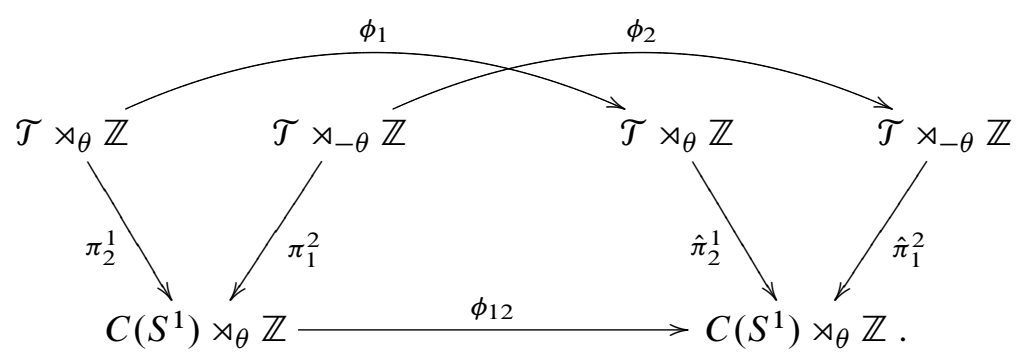

Here isomorphisms $\phi$ are given on respective generators by

$$
z \mapsto z u, \quad u \mapsto u
$$

The $\mathrm{C}^{*}$-subalgebra of $\mathrm{U}(1)$-invariants is the $\mathrm{C}^{*}$-algebra of the mirror quantum 2-sphere from [HMS06b]. As mentioned in Subsection 2.5, we can pass from the $\mathrm{U}(1)-\mathrm{C}^{*}$-algebra $C\left(S_{p q \theta}^{3}\right)$ to the associated principal comodule algebra, and this procedure always commutes with taking fibre products. (This is unlike the $\mathrm{C}^{*}$ completion procedure that needs an additional assumption to commute with taking fibre products, e.g., see $[\mathrm{HW}]$.) In this way, we obtain a subalgebra $P \subseteq C\left(S_{p q \theta}^{3}\right)$ which is a piecewise trivial $\mathbb{C} \mathbb{Z}$-comodule algebra, so that it fits the setting of this paper. The invariant subalgebra $P^{\mathrm{co} H}$ is again the $\mathrm{C}^{*}$-algebra of the mirror quantum 2-sphere.

There is another natural Hopf-like U(1)-action on $C\left(S_{p q \theta}^{3}\right)$ described in [HMS06b]. Again, its restriction to the two crossed products making up the fibre product $C\left(S_{p q \theta}^{3}\right)$ is not the canonical action of $\mathrm{U}(1)$. To transform this fibre product into an isomorphic one but carrying the canonical U(1)-action on the crossed products, we take the fibre product defined by the morphisms

$$
\begin{aligned}
& \check{\pi}_{2}^{1}: \mathcal{T} \rtimes_{-\theta} \mathbb{Z} \rightarrow C\left(S^{1}\right) \rtimes_{-\theta} \mathbb{Z}, \quad z_{-} \mapsto Z_{-}, \quad u_{-} \mapsto U_{-}, \\
& \check{\pi}_{1}^{2}: \mathcal{T} \rtimes_{-\theta} \mathbb{Z} \rightarrow C\left(S^{1}\right) \rtimes_{-\theta} \mathbb{Z}, \quad z_{-} \mapsto Z_{-}, \quad u_{-} \mapsto Z_{-} U_{-} .
\end{aligned}
$$

Here $Z_{-}$and $U_{-}$are the generators of $C\left(S^{1}\right) \rtimes_{-\theta} \mathbb{Z}$ corresponding to $C\left(S^{1}\right)$ and $\mathbb{Z}$ respectively. The identifying maps are now given by

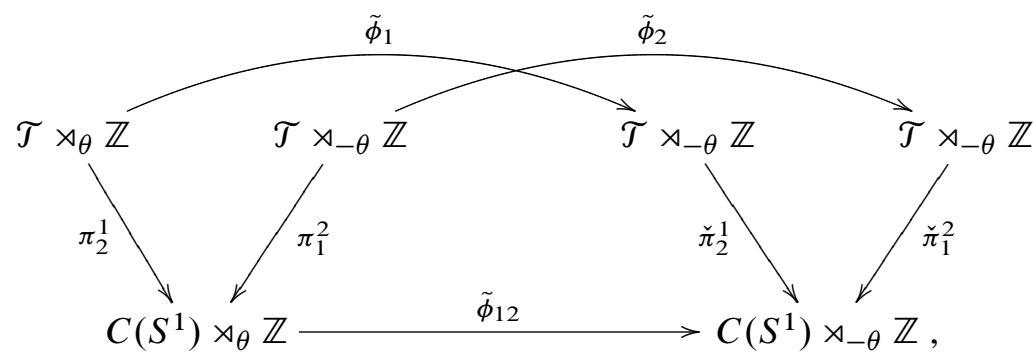




$$
\begin{array}{cl}
\tilde{\phi}_{1}: z_{+} \mapsto z_{-} u_{-}, & u_{+} \mapsto u_{-}^{-1}, \\
\tilde{\phi}_{2}: z_{-} \mapsto u_{-}^{-1} z_{-}, & u_{-} \mapsto u_{-}, \\
\tilde{\phi}_{12}: Z_{+} \mapsto Z_{-} U_{-}, & U_{+} \mapsto U_{-}^{-1} .
\end{array}
$$

The subalgebra of U(1)-invariants is now the $\mathrm{C}^{*}$-algebra of a generic Podleś quantum 2-sphere [P-P87]. Note that it is not possible to obtain in this way the polynomial algebra of a generic Podleś sphere - replacing $\mathcal{T}=P_{i}^{\operatorname{co} H}$ by the polynomial algebra of a quantum disc that is generated by $x$ satisfying $x^{*} x-q x x^{*}=1-q$ would yield a non-isomorphic algebra [CM00]. This is related to the fact that already in the commutative setting the algebra of polynomial functions on a sphere has no covering corresponding to two hemispheres because there are no nontrivial polynomials vanishing on a hemisphere.

Acknowledgements. The authors are particularly indebted to Tomasz Maszczyk and Fred Van Oystaeyen for their help with lattices. They are also very grateful to the referee for his/her effort to bring this paper to its final form, to Paweł Witkowski for producing the join picture, and to Paul F. Baum, Gabriella Böhm, Tomasz Brzeziński, Nigel Higson, George Janelidze, Max Karoubi, Dorota Marciniak, Ryszard Nest, Marc A. Rieffel, Marcin Szamotulski, Joseph C. Várilly and Elmar Wagner for discussions. This work was partially supported by the European Commission grants RITA-CT-2004-505493 (PMH), EIF-515144 (UK), MKTD-CT-2004-509794 (RM), the Polish Government grants 1 P03A 03626 (PMH, RM), 115/E-343/SPB/6.PR UE/DIE 50/2005-2008 (PMH, BZ), and the University of Łódź Grant 795 (BZ). PMH thanks the Max Planck Institute for Mathematics in the Sciences in Leipzig and the Institut des Hautes Études Scientifiques for their hospitality and financial support. Finally, PMH expresses his deepest gratitude to Hans-Jürgen Schneider for his untiring guidance in Hopf-Galois theory.

\section{References}

[BS93] S. Baaj and G. Skandalis, Unitaires multiplicatifs et dualité pour les produits croisés de $C^{*}$-algèbres. Ann. Sci. École Norm. Sup. (4) 26 (1993), 425-488. Zbl 0804.46078 MR 1235438

[BH] P. F. Baum and P. M. Hajac, The Peter-Weyl-Galois theory of compact principal bundles. In preparation.

[BHMS05] P. F. Baum, P. M. Hajac, R. Matthes, and W. Szymański, The $K$-theory of Heegaard-type quantum 3-spheres. K-Theory 35 (2005), 159-186; erratum ibid. 37 (2006), 211. Zbl 1111.46051 Zbl 1210.46054 MR 2240219

[BHMS] P. F. Baum, P. M. Hajac, R. Matthes, W. Szymański, Noncommutative geometry approach to principal and associated bundles. In Quantum symmetry in noncommutative geometry, ed. P. M. Hajac, European Math. Soc., to appear. 
[B-G67] G. Birkhoff, Lattice theory. 3rd ed., Amer. Math. Soc. Colloq. Publ. 25, Amer. Math. Soc., Providence, R.I., 1967. Zbl 0153.02501 MR 0227053

[BM89] R. J. Blattner and S. Montgomery, Crossed products and Galois extensions of Hopf algebras. Pacific J. Math. 137 (1989), 37-54. Zbl 0675.16017 MR 983327

[B-FP95] F. P. Boca, Ergodic actions of compact matrix pseudogroups on $C^{*}$-algebras. Astérisque 232 (1995), 93-109. Zbl 0842.46039 MR 1372527

[BB05] G. Böhm and T. Brzeziński, Strong connections and the relative Chern-Galois character for corings. Internat. Math. Res. Notices (2005), 2579-2625. Zbl 1111.16038 MR 2182707

[B-GE93] G. E. Bredon, Topology and geometry. Graduate Texts in Math. 139, SpringerVerlag, New York 1993. Zbl 0791.55001 MR 1224675

[BH04] T. Brzeziński and P. M. Hajac, The Chern-Galois character. C. R. Math. Acad. Sci. Paris 338 (2004), 113-116. Zbl 1061.16037 MR 2038278

[BK96] R. J. Budzyński and W. Kondracki, Quantum principal fibre bundles: topological aspects. Rep. Math. Phys. 37 (1996), 365-385. Zbl 0886.58004 MR 1403770

[CM00] D. Calow and R. Matthes, Covering and gluing of algebras and differential algebras. J. Geom. Phys. 32 (2000), 364-396. Zbl 0969.46051 MR 1734767

[CM02] D. Calow and R. Matthes, Connections on locally trivial quantum principal fibre bundles. J. Geom. Phys. 41 (2002), 114-165. Zbl 1026.46064 MR 1872383

[CKP93] A. Carboni, G. M. Kelly, and M. C. Pedicchio, Some remarks on Maltsev and Goursat categories. Appl. Categ. Structures 1 (1993), 385-421. Zbl 0799.18002 MR 1268510

[DGH01] L. Dąbrowski, H. Grosse, and P. M. Hajac, Strong connections and Chern-Connes pairing in the Hopf-Galois theory. Comm. Math. Phys. 220 (2001), 301-331. Zbl 0990.58008 MR 1844628

[DHH] L. Dąbrowski, T. Hadfield, and P. M. Hajac, A noncommutative join construction. In preparation.

[D-R97] R. Dedekind, Über Zerlegungen von Zahlen durch ihre größten gemeinsamen Teiler. In Fest-schrift der Herzoglichen Technischen Hochschule CaroloWilhelmina dargeboten den naturwissenschaftlichen Theilnehmern an der 69. Versammlung deutscher Naturforscher und Ärzte vom Herzog. braunschw. Staatsministerium, Friedr. Vieweg \& Sohn, Braunschweig 1897; Gesammelte mathematische Werke, Vol. 2, 103-147, Friedr. Vieweg \& Sohn, Braunschweig 1931. JFM 28.0186.04

[D-M97] M. Durđević, Quantum principal bundles and corresponding gauge theories. $J$. Phys. A 30 (1997), 2027-2054. Zbl 0928.46051 MR 1450313

[GN43] I. Gelfand and M. Neumark, On the imbedding of normed rings into the ring of operators in Hilbert space. Mat. Sb. (N.S.) 12 (54) (1943), 197-213. Zbl 0060.27006 MR 0009426

[H-PM96] P. M. Hajac, Strong connections on quantum principal bundles. Comm. Math. Phys. 182 (1996), 579-617. Zbl 0873.58007 MR 1461943

[HMS06a] P. M. Hajac, R. Matthes, and W. Szymanski, A locally trivial quantum Hopf fibration. Algebr. Represent. Theory 9 (2006), 121-146. Zbl 1132.58007 MR 2238363 
[HMS06b] P. M. Hajac, R. Matthes, and W. Szymański, Noncommutative index theory for mirror quantum spheres. C. R. Math. Acad. Sci. Paris 343 (2006), 731-736. Zbl 1114.46052 MR 2284701

[HW] P. M. Hajac and E. Wagner, The pullbacks of principal coactions. Preprint 2009. arXiv: 1001.0075

[KS97] A. Klimyk and K. Schmüdgen, Quantum groups and their representations. Texts Monographs Phys., Springer-Verlag, Berlin 1997. Zbl 0891.17010 MR 1492989

[M-S93] S. Montgomery, Hopf algebras and their actions on rings, CBMS Regional Conf. Ser. in Math. 82, Amer. Math. Soc., Providende, RI, 1993. Zbl 0793.16029 MR 1243637

[P-RS61] R. S. Palais, On the existence of slices for actions of non-compact Lie groups. Ann. of Math. (2) 73 (1961), 295-323. Zbl 0103.01802 MR 0126506

[P-GK99] G. K. Pedersen, Pullback and pushout constructions in $C^{*}$-algebra theory. $J$. Funct. Anal. 167 (1999), 243-344. Zbl 0944.46063 MR 1716199

[P-MJ94] M. J. Pflaum, Quantum groups on fibre bundles. Comm. Math. Phys. 166 (1994), 279-315. Zbl 0824.58009 MR 1309551

[P-P87] P. Podleś, Quantum spheres. Lett. Math. Phys. 14 (1987), 193-202. Zbl 0634.46054 MR 919322

[P-P95] P. Podleś, Symmetries of quantum spaces. Subgroups and quotient spaces of quantum $S U(2)$ and $S O(3)$ groups. Comm. Math. Phys. 170 (1995), 1-20. Zbl 0853.46074 MR 1331688

[R-GC97] G.-C. Rota, The many lives of lattice theory. Notices Amer. Math. Soc. 44 (1997), 1440-1445. Zbl 0908.06001 MR 1488572

[SS58] P. Samuel and O. Zariski, Commutative algebra. Vol. I, D. van Nostrand, Princeton 1958. Zbl 0081.26501 MR 0090581

[SS05] P. Schauenburg and H.-J. Schneider, On generalized Hopf Galois extensions. J. Pure Appl. Algebra 202 (2005), 168-194. Zbl 1081.16045 MR 2163407

[SS] P. Schauenburg and H.-J. Schneider, Galois-type extensions and Hopf algebras. In Quantum symmetry in noncommutative geometry, ed. P. M. Hajac, European Math. Soc., to appear.

[S-HJ94] H.-J. Schneider, Hopf Galois extensions, crossed products, and Clifford theory. In Advances in Hopf algebras, Lecture Notes in Pure and Appl. Math. 158, Marcel Dekker, New York 1994, 267-297. Zbl 0817.16017 MR 1289428

[S-PM] P. M. Sołtan, On actions of compact quantum groups. Illinois J. Math., to appear. Preprint 2010. arXiv: 1003.5526

[W-SL87] S. L. Woronowicz, Compact matrix pseudogroups. Comm. Math. Phys. 111 (1987), 613-665. Zbl 0627.58034 MR 901157

[W-SL98] S. L. Woronowicz, Compact quantum groups. In Symétries quantiques (Les Houches, 1995), North-Holland, Amsterdam 1998, 845-884. Zbl 0997.4645 MR 1616348

[Y-K54] K. Yamamoto, Logarithmic order of free distributive lattice. J. Math. Soc. Japan 6 (1954), 343-353. Zbl 0056.26301 MR 0067086 
Received March 26, 2010; revised May 18, 2010

P. M. Hajac, Instytut Matematyczny, Polska Akademia Nauk, ul. Śniadeckich 8, Warszawa, 00-956 Poland, and Katedra Metod Matematycznych Fizyki, Uniwersytet Warszawski, ul. Hoża 74, Warszawa, 00-682 Poland

E-mail: pmh@impan.pl

U. Krähmer, University of Glasgow, Department of Mathematics, University Gardens, G12 8QW, Scotland, and Instytut Matematyczny, Polska Akademia Nauk, ul. Śniadeckich 8, Warszawa, 00-956 Poland

E-mail: ukraehmer@maths.gla.ac.uk

R. Matthes, Katedra Metod Matematycznych Fizyki, Uniwersytet Warszawski, ul. Hoża 74, Warszawa, 00-682 Poland

E-mail: matthes@fuw.edu.pl

B. Zieliński, Instytut Matematyczny, Polska Akademia Nauk, ul. Śniadeckich 8, Warszawa, 00-956 Poland, and Katedra Fizyki Teoretycznej i Informatyki, Uniwersytet Łódzki, Pomorska 149/153, 90-236 Łódź, Poland

E-mail: bzielinski@uni.lodz.pl 